\title{
Modeling the Dynamics of Shanghai Interbank Offered Rate Based on Single-Factor Short Rate Processes
}

\author{
Xili Zhang \\ School of Management, Zhejiang University, Hangzhou 310058, China \\ Correspondence should be addressed to Xili Zhang; zhangxili831@gmail.com \\ Received 13 February 2014; Accepted 22 March 2014; Published 16 April 2014 \\ Academic Editor: Weiguo Zhang \\ Copyright ( 2014 Xili Zhang. This is an open access article distributed under the Creative Commons Attribution License, which \\ permits unrestricted use, distribution, and reproduction in any medium, provided the original work is properly cited. \\ Using the Shanghai Interbank Offered Rate data of overnight, 1 week, 2 week and 1 month, this paper provides a comparative analysis \\ of some popular one-factor short rate models, including the Merton model, the geometric Brownian model, the Vasicek model, \\ the Cox-Ingersoll-Ross model, and the mean-reversion jump-diffusion model. The parameter estimation and the model selection \\ of these single-factor short interest rate models are investigated. We document that the most successful model in capturing the \\ Shanghai Interbank Offered Rate is the mean-reversion jump-diffusion model.
}

\section{Introduction}

The short-term interest rate is not only the fundamental importance to the management of interest rate risk, but also plays an important role in many areas of asset pricing studies. Thus it is important to understand and model the term structure of interest rates. In fact, there are many benefits from a better understanding of the short-term structure of interest rates and the models associated with it. Historically, many popular models currently used by academic researchers and practitioners have been developed in a continuous-time setting, which provides a rich framework for specifying the dynamic behavior of the short-term riskless rate. Among the existing models, one-factor models are a popular class of interest rate models which are used for these purposes, especially in the pricing of interest rate derivatives.

While the Black and Scholes [1] has rapidly established the model for stock prices, a large number of continuous time approaches are simultaneously used among academics and practitioners in the field of interest rates contingent claims. Originating from Merton [2], who suggested using the diffusion process to model the short-term interest rate, many economists, econometricians, and mathematicians spent much efforts modeling the short-term structure of interest rates both in continues time and discrete time. From historical data of interest rate, Vasicek [3] observed that short-term interest rate would revert to a certain level in a long run and suggested to use Ornstein-Uhlenbeck process to model the mean reversion property of interest rate. Then Cox and Ross [4] showed that the volatility of interest rate increased with the level of interest rate and presented the Cox-Ingersoll-Ross model. Incorporating the mean reversion, level effect, and stationarity, Chan et al. [5] constructed a general model of short-term interest rate, which generalized all single-factor diffusion models. Furthermore, Aitt-Sahalia [6] found the possibility of nonlinear drift in the case of Eurodollar rates. No matter which parameters we put in the drift component or random component, all the models mentioned above are all continuous models. In fact, a large number of empirical studies have demonstrated that these continuous models do not adequately explain the observed characteristics of interest rates (see, e.g., [7-9]). In other words, the empirical distribution of daily log returns of interest rates differs in many ways from the diffusion process. First of all, the actual $\log$ returns are characterized by large fluctuations in value, such as crashes and rallies. Besides, the empirical distribution of log returns is asymmetric (mostly negatively skewed) and leptokurtic. To capture large fluctuations, asymmetric and leptokurtic, of log returns on interest rates, we introduce the compound poisson process to the mean-reverting process based on the ideas of Merton [10], Ball and Torous [11], and Kou [12]. Since various economic shocks, news announcements, and government interventions in bond markets have pronounced effects on the behavior of spot interest rates and 
tend to generate jumps in interest rate data, it is natural to capture the excessive kurtosis and heavy tails of interest rates using jump-diffusion models. Consequently, to capture jumps in trend of spot interest rates, the mean reverting jump diffusion model will be employed in this paper.

Although the spot rate dynamics have been extensively examined in mature markets in the literature, there has been little study of interest rates in China and other emerging markets. As stated in Hong et al. [13], the reason for this situation is due to the relatively short history of the Chinese bond markets and the strict regulation of Chinese interest rates. It is well known that China's market-oriented interest rate consists of the following components: the first is Renminbi credit Lending rate, which includes IBO001, IBO007, IBO01M, IBO03M, IBO04M, IBO06M, IBO09M, and IBO01Y. The second component is Shanghai Interbank Offered Rate (hereafter SHIBOR), which includes Sro/n, Srlw, Sr2w, Sr3m, Sr6m, Sr9m, and Srly. The third one is the interbank bond repurchase rate, which includes the R001, R007, R014, R021, R1M, R2M, R3M, R4M, R6M, R9M, and $\mathrm{R} 1 \mathrm{Y}$. The forth component is the exchange bond repurchase rate. In fact, the most commonly used is SHIBOR. Hence, this paper devotes to describing the behavior of SHIBOR. It is worth emphasizing that capturing the characteristics of Chinese interest rates and constructing corresponding term structures are important not only for developing efficient financial markets, but also for pricing interest-rate derivative. Moreover, the interest rate is one of the fundamental macroeconomic indicators in China. In fact, Chinese interest rate is fundamental for pricing of bonds and other debt instruments such as bank loans, mortgages, corporate debts, and interest rate derivatives. This is particularly important now for China; considering the tremendous efforts the authorities have been taking in developing an active Renminbi bond market. It is also a useful tool for portfolio evaluation, risk management, and monetary policy analysis. For example, it can help policy makers gauge market expectations of future interest rates and inflation rates (see [14]).

Obviously, it is well known that Chinese interest rates are quite heavily managed by the authorities. For example, it is often found that Chinese interest rates are strongly subject to administrative control by the government, and their mechanism is quite different from that of other developed markets (see [13]). Consequently, the models popular in mature markets may do not work for the Chinese market. Hence studying the term structure of Chinese interest rates has attracted much interest. Researchers in economics, statistical physics, and probability have approached the problem from different perspectives and proposed various models. In particular, one of the key points in this area is how these existing models compare in terms of their ability to capture the actual behavior of the Chinese interest rates. In order to solve the problems raised by the model selection, in this paper, we provide a comprehensive empirical study on the dynamics of Chinese interest rates. Particularly, using maximum likelihood estimation and SHIBOR data, we estimate and compare a variety of single-factor models, including the Merton model, the geometric Brownian motion, the Vasicek model, the Cox-Ingersoll-Ross model, and the mean-reverting jump diffusion model. Since the models considered in this paper belong to "separate" families of distributions, the models under consideration are "nonnested." Consequently, we use the Vuong test (see [15]) to test the adequacy of these models for Chinese interest rates. The empirical results are clearly in favor of the mean-reverting jump diffusion model. The reason for this is that the mean-reversion jump-diffusion model combines a continuous Poisson jump component, with a continuous log normally distributed component.

The structure of this paper is organized as follows. In Section 2, we give an overview of the short-term interest rate models examined in the paper. Both the parameter estimation methods for these proposed models and the model selection criterion are introduced in Section 3. In Section 4, we review the history of the Chinese interest rate liberalization and describe the data on Chinese interest rates. The empirical results of parameter estimation and model selection are also provided in the remaining part of Section 4 . Section 5 summarizes the paper and makes concluding remarks.

\section{Spot Interest Rate Models}

Understanding and modeling the dynamics of Chinese interest rates represents one of the most challenging topics of financial research. In the literature, one-factor models are a popular class of interest rate models which are used for pricing interest rate derivatives. In fact, a commonly studied problem in finance is the modeling of the dynamics of the shortterm riskless interest rate, often using the following general stochastic differential equation:

$$
d r_{t}=\mu\left(t, r_{t}\right) d t+\sigma\left(t, r_{t}\right) d B_{t}, \quad t \geq 0,
$$

where $r_{t}$ is the modeled interest rate, $\mu\left(t, r_{t}\right)$ and $\sigma\left(t, r_{t}\right)$ are the drift and the diffusion term of the interest rate process, respectively, and $\left(B_{t}, t \geq 0\right)$ is a Brownian motion or a Wiener process.

The stochastic differential equation given in (1) defines a broad class of interest rate processes which includes many well-known interest rate models. For example, one can obtain some alternative models by allowing the drift function to have a zero, linear, and nonlinear specifications and allowing the diffusion function to be a constant or to depend on the interest rate level, which is referred to as the "level effect." In the literature, researchers have tried to propose better models that have the capability to fit the data more efficiently and can be used for pricing the interest rate derivatives. Now, some models, which are considered in this paper, will be introduced.

2.1. Merton Model. Merton [2] was the first one to propose a general stochastic process as a model for the short rate. This model is defined as

$$
d r_{t}=\mu d t+\sigma d B_{t}, \quad t \geq 0,
$$

where $\mu$ and $\sigma$ are some constants, and $\left(B_{t}, t \geq 0\right)$ is a Brownian motion or a Wiener process. 
2.2. Geometric Brownian Motion. Inspired by the research of Black and Scholes [1], Marsh and Rosenfeld [16] introduce the geometric Brownian motion (hereafter GBM) to capture the dynamics of interest rates

$$
d r_{t}=\mu r_{t} d t+\sigma r_{t} d B_{t}, \quad t \geq 0,
$$

where $\mu$ and $\sigma$ are some constants, and $\left(B_{t}, t \geq 0\right)$ is a Brownian motion or a Wiener process.

Since the short rate $r_{t}$ in the GBM is lognormally distributed, it is always positive. A disadvantage of the GBM is that $r_{t}$ is mean reverting if and only if $\mu<0$, and then the mean reversion level is zero.

2.3. Vasicek Model. Despite the popularity of GBM, some scholars have examined the empirical evidence for meanreverting behavior in interest rates. Indeed, there are also compelling economic arguments in favor of mean reversion. When the rates are high, the economy tends to slow down and borrowers require less funds. Furthermore, the rates pull back to its equilibrium value and the rates decline. On the contrary when the rates are low, there tends to be high demand for funds on the part of the borrowers and rates tend to increase. This feature is particularly attractive without it, interest rates could drift permanently upward the way stock prices do, and this is simply not observed in practice. To capture this mean reversion of interest rates, Vasicek [3] assumed that the instantaneous spot rate evolves as an Orstein-Uhlenbeck process with constant coefficients. This process is defined as

$$
d r_{t}=\mu\left(\theta-r_{t}\right) d t+\sigma d B_{t}, \quad t \geq 0,
$$

where $\theta, \mu$, and $\sigma$ are positive constants, and $\left(B_{t}, t \geq 0\right)$ is a Brownian motion or a Wiener process.

Let us mention that the Vasicek model was the first one to capture mean reversion, which is an essential characteristic of the interest rate. In fact, the Vasicek model is a so called "mean reverting process." When $r_{t}>\theta$, the drift is negative; while $r_{t}<\theta$, the drift is positive. Hence the drift is always directed to $\theta$, and that may thus be interpreted as an "equilibrium level" or "long run mean" of the short rate $r_{t}$. The parameter $\mu$ represents then the "strength" of this mean reversion, letting $\mu \rightarrow 0^{+}$correspond to "turning off" the mean reversion effect.

2.4. Cox-Ingersoll-Ross Model. The major drawback of the Vasicek model is that the short rate $r_{t}$, for each time $t$, can be negative with positive probability. This is a major drawback of the Vasicek model. In order to overcome this problem, the Cox-Ingersoll-Ross (hereafter CIR) model came through.

The CIR model is the square root process that is proposed by Cox and Ross [4]. This model has also been used extensively in developing valuation models for interest rate contingent claims. This model is expressed as

$$
d r_{t}=\mu\left(\theta-r_{t}\right) d t+\sigma \sqrt{r_{t}} d B_{t}, \quad t \geq 0
$$

where $\theta, \mu$, and $\sigma$ are positive constants, and $\left(B_{t}, t \geq 0\right)$ is a Brownian motion or a Wiener process.
The process followed by the short rate in the CIR model is also called a square-root process. The good mean reversion property in the Vasicek model is preserved in the CIR model. The bad property of possible negativity in the Vasicek model is removed in the CIR model under some assumptions and hence ensuring that the origin is inaccessible to the process. On the other hand, the distribution of the short rate in the CIR model is neither normal nor lognormal but it possesses a noncentral Chi-squared distribution.

2.5. Mean-Reverting Jump Diffusion Model. Since models of interest rates mentioned above are pure diffusion processes, the proposed dynamics of the various short interest rates do not meet empirical evidence on the presence of discontinuities in the process of the interest rate. The pure diffusion processes may not be sufficient to capture all of the observed asymmetry in interest rate changes. Furthermore, various economic shocks, news announcements, and government interventions in bond markets have pronounced effects on the behavior of interest rates and tend to generate large jumps in interest rate data. Statistically many researchers have shown that diffusion models (even with stochastic volatility) cannot generate the excessive leptokurtosis exhibited by the changes of the spot rates and that jump-diffusion models are a convenient way to generate excessive kurtosis or, more generally, heavy tails. For example, Das [7] extended the Vasicek [3] model to a jump diffusion model and showed that incorporating jumps could capture many empirical features of the Fed Fund rate, which cannot be explained by the continuous diffusion models. Johannes [9] and Lim et al. [17] provided evidence of the presence of jumps in the dynamics of interest rates. Consequently, to capture the randomly occurring "jumps," it is natural to use the mean-reversion jump-diffusion (hereafter MRJD) model to describe interest rates, which can be expressed as

$$
d r_{t}=\mu\left(\theta-r_{t}\right) d t+\sigma d B_{t}+J d q_{t}, \quad t \geq 0,
$$

where $\theta, \mu$, and $\sigma$ are positive constants, and the Brownian motion $B_{t}$ is responsible for small fluctuations around the long-term mean $\theta$, while an independent compound Poisson process $q_{t}$ produces infrequent but large jumps of size $J$. Moreover, the jump size $J$ is assumed to be normally distributed with mean $\mu_{J}$ and standard deviation $\sigma_{J}$. The arrival of a jump is governed by a Poisson increment $d q_{t}$ with jump intensity $\lambda$, denoted as the number of jumps occurring on a particular period.

We can see that the second random term in (6) represents the jump component, which allows us to incorporate sudden market reaction due to unforeseen events. If the mean size of the interest rate jump is negative, then it means that on average the fall in interest rates tends to be bigger than the subsequent rise. In fact, the proposed jump component of the model (6) is inspired by Merton [10], who used this very popular process to describe the evolution of stocks, commodity prices, and indices. 


\section{The Econometric Approach}

We would like to mention that the models proposed in this paper are continuous stochastic processes. One can perform statistical inference of these models based on either one or many realizations of the process over a time period. However, in practice, it is virtually impossible to observe a process continuously over any given time period, for example, due to limitations on the precision of the measuring instruments or due to the unavailability of observations at every time point. One often encounters practical difficulties to obtain a complete continuous observation of the sample path, and only the discrete time observations are possible. In this section, we will discuss the problem of estimating unknown parameters in the models proposed in this paper based on sampled data. Moreover, we will also introduce the likelihood ratio test in the latter part of this section.

3.1. Estimation Procedures. In applications usually the processes cannot be observed continuously. Only discrete time observations are available. Hence, in this section, from discrete observations, we describe the econometric approach used in estimating the parameters of these interest rate models proposed in this paper. In order to obtain the unknown parameters in these models mentioned in this paper, we should choose a suitable method to estimate the unknown parameters. In the literature, several heuristic methods are available for solving problems of this sort. The most popular approaches are either the maximum likelihood estimation or the least squares estimation. In this section, we will adopt the maximum likelihood approach. The reasons for choosing this method are threefold. First, this technique has been applied efficiently in a large set (see, e.g., [18]). The second reason is its well-documented favorable properties, such as being asymptotically consistent, unbiased, efficient, and normally distributed over the true parameter values (see, e.g., [19]). The third reason is that some of these proposed models provide the exact maximum likelihood estimator (hereafter MLE).

To implement the maximum likelihood approach, first we need to define the likelihood function. Then we can obtain the parameter values that maximize the value of this likelihood function. Without loss of generality, we assume that the process of $r_{t}$ is observed at discrete-time instants $\left(t_{1}, t_{2}, \ldots, t_{N}\right)$. Thus the observation vector is $\mathbf{r}=\left(r_{t_{1}}, r_{t_{2}}, \ldots, r_{t_{N}}\right)^{\prime}$, where the prime' is used to denote the vector transposition and all the nonprimed vectors are row vectors. In particular, to simplify notations we assume $t_{k}=k h, k=1,2, \ldots, N$ for a fixed-step size $h>0$. General deterministic observation times $t_{k}$ can be also considered in a similar way. Thus, throughout the paper the discrete-time observation can be expressed in the form of vector as $\mathbf{r}=\left(r_{h}, r_{2 h}, \ldots, r_{N h}\right)^{\prime}$.

3.1.1. MLE for Merton Model. Now, we focus on the maximum likelihood estimation for Merton model. Using ItôDoeblin formula and setting $B_{0}=0$, we can obtain the solution of (2) as

$$
r_{t}=\mu t+\sigma B_{t}, \quad t \geq 0
$$

Thus, the discrete-time observation can be expressed in the form of vector as

$$
\mathbf{r}=\mu \mathbf{t}+\sigma \mathbf{B}_{t}
$$

where $\mathbf{r}=\left(r_{h}, r_{2 h}, \ldots, r_{N h}\right)^{\prime}, \mathbf{t}=(h, 2 h, \ldots, N h)^{\prime}$, and $\mathbf{B}_{t}=$ $\left(B_{h}, B_{2 h}, \ldots, B_{N h}\right)^{\prime}$.

Inspired by $\mathrm{Hu}$ et al. [19], we can obtain the exact maximum likelihood estimators of $\mu$ and $\sigma^{2}$. As the law of $\mathbf{r}$ is Gaussian, the joint probability density function of $\mathbf{r}$ can be written as

$$
g(\mathbf{r})=\left(2 \pi \sigma^{2}\right)^{-N / 2}|\Gamma|^{-1 / 2} \exp \left(-\frac{1}{2 \sigma^{2}}(\mathbf{r}-\mu \mathbf{t})^{\prime} \Gamma^{-1}(\mathbf{r}-\mu \mathbf{t})\right),
$$

where

$$
\Gamma=\left[\operatorname{Cov}\left[B_{i h}, B_{j h}\right]\right]_{i, j=1,2, \ldots, N}=h(i \wedge j)_{i, j=1,2, \ldots, N} .
$$

The MLEs of $\mu$ and $\sigma^{2}$ from the observation $\mathbf{r}$ are given by

$$
\begin{aligned}
\widehat{\mu} & =\frac{\mathbf{t}^{\prime} \Gamma^{-1} \mathbf{r}}{\mathbf{t}^{\prime} \Gamma^{-1} \mathbf{t}}, \\
\widehat{\sigma}^{2} & =\frac{1}{N} \frac{\left(\mathbf{r}^{\prime} \Gamma^{-1} \mathbf{r}\right)\left(\mathbf{t}^{\prime} \Gamma^{-1} \mathbf{t}\right)-\left(\mathbf{t}^{\prime} \Gamma^{-1} \mathbf{r}\right)^{2}}{\mathbf{t}^{\prime} \Gamma^{-1} \mathbf{t}} .
\end{aligned}
$$

3.1.2. MLE for GBM. It is well known that the discretized version of (3) can be written as

$$
\begin{aligned}
r_{(i+1) h}-r_{i h} & =\mu r_{i h} h+\sigma r_{i h}\left(B_{(i+1) h}-B_{i h}\right) \\
& =\mu r_{i h} h+r_{i h} \epsilon_{i+1}, \quad t \geq 0,
\end{aligned}
$$

where $\epsilon_{i+1}$ is an i.i.d $\mathcal{N}\left(0, \sigma^{2} h\right)$ random variate.

It is easy to check that the MLEs of $\mu$ and $\sigma^{2}$ are given by

$$
\begin{gathered}
\widehat{\mu}=\frac{1}{N h} \sum_{i=1}^{N}\left(\frac{r_{i}}{r_{i-1}}-1\right), \\
\widehat{\sigma}^{2}=\frac{1}{N h} \sum_{i=1}^{N}\left(\frac{r_{i}}{r_{i-1}}-1-\widehat{\mu} h\right)^{2} .
\end{gathered}
$$

3.1.3. MLE for Vasicek Model. Using Itô-Doeblin formula, for any $t \geq s$, we can obtain the solution of (4)

$$
r_{t}=\theta+\left(r_{s}-\theta\right) e^{-\mu(t-s)}+\sigma \int_{s}^{t} e^{-\mu(t-s)} d B_{u}, \quad r_{0}=0 .
$$

Moreover, for $s<t$, given the set of information at time $s, r_{t}$ is normally distributed

$$
r_{t} \mid \mathscr{F}_{s} \sim \mathcal{N}\left(\theta+\left(r_{s}-\theta\right) e^{-\mu(t-s)}, \frac{\sigma^{2}}{2 \mu}\left(1-e^{-2 \mu(t-s)}\right)\right) .
$$


Now, the probability density function of the process (4) can be written as

$$
\begin{aligned}
& p\left(r_{i+1} \mid r_{i} ; \mu, \theta, \widetilde{\sigma}\right) \\
& \quad=\frac{1}{\sqrt{2 \pi \widetilde{\sigma}^{2}}} \exp \left[-\frac{\left(r_{i}-\theta-\left(r_{i-1}-\theta\right) e^{-\mu h}\right)^{2}}{2 \widetilde{\sigma}^{2}}\right],
\end{aligned}
$$

with $\widetilde{\sigma}^{2}=\left(\left(1-e^{-2 \mu h}\right) / 2 \mu\right) \sigma^{2}$.

Without loss of generality, the log-likelihood function of a set of observation can be derived from the conditional density function:

$$
\begin{aligned}
L(\mu, \theta, \widetilde{\sigma})= & -\frac{N}{2} \ln (2 \pi)-N \ln (\widetilde{\sigma}) \\
& -\frac{1}{2 \widetilde{\sigma}^{2}} \sum_{i=1}^{N}\left[r_{i}-\theta-\left(r_{i-1}-\theta\right) e^{-\mu h}\right]^{2} .
\end{aligned}
$$

Then we can derive the MLEs for $\mu, \theta$, and $\tilde{\sigma}^{2}$, by maximizing (17) with respect to $\mu, \theta$, and $\tilde{\sigma}^{2}$. A standard calculation yields

$$
\begin{aligned}
\hat{\theta}= & \frac{S_{y} S_{x x}-S_{x} S_{x y}}{N\left(S_{x x}-S_{x y}\right)-\left(S_{x}^{2}-S_{x} S_{y}\right)}, \\
\widehat{\mu}= & -\frac{1}{h} \ln \frac{S_{x y}-\widehat{\theta} S_{x}-\widehat{\theta} S_{y}+N \widehat{\theta}^{2}}{S_{x x}-2 \widehat{\theta} S_{x}+N \hat{\theta}^{2}}, \\
\widehat{\sigma}^{2}= & \frac{2 \widehat{\mu}}{N\left(1-e^{-2 \hat{\mu} h}\right)} \\
& \times\left[S_{y y}-2 e^{-\widehat{\mu} h} S_{x y}+e^{-2 \widehat{\mu} h} S_{x x}\right. \\
& -2 \widehat{\theta}\left(1-e^{-\widehat{\mu} h}\right)\left(S_{y}-e^{-\widehat{\mu} h} S_{x}\right) \\
& \left.+N \widehat{\theta}^{2}\left(1-e^{-\widehat{\mu} h}\right)^{2}\right],
\end{aligned}
$$

where $S_{x}=\sum_{i=1}^{N} r_{i-1}, S_{y}=\sum_{i=1}^{N} r_{i}, S_{x x}=\sum_{i=1}^{N} r_{i-1}^{2}, S_{x y}=$ $\sum_{i=1}^{N} r_{i-1} r_{i}$, and $S_{y y}=\sum_{i=1}^{N} r_{i}^{2}$.

3.1.4. MLE for CIR Model. In mathematical finance, the CIR model describes the evolution of interest rates. Actually, if $\mu$, $\theta$, and $\sigma$ are all positive and $2 \mu \theta \sigma^{2}$ holds, the CIR process is well defined and has a steady state distribution. The transition density of the CIR model has been originally derived in [20], which is expressed as

$$
p\left(r_{i+1} \mid r_{i} ; \mu, \theta, \widetilde{\sigma}\right)=c e^{-u_{i}-v_{i+1}}\left(\frac{v_{i+1}}{u_{i}}\right)^{q / 2} I_{q}\left(2 \sqrt{u_{i} v_{i+1}}\right)
$$

where $c=2 \mu / \sigma^{2}\left(1-e^{-\mu h}\right), u_{i}=c r_{i} e^{-\mu h}, v_{i+1}=c r_{i+1}, q=$ $2 \mu \theta / \sigma^{2}-1$, and $I_{q}\left(2 \sqrt{u_{i} v_{i+1}}\right)$ is the modified Bessel function of the first kind and of the order $q$.
Thus we can easily derive the log-likelihood function:

$$
\begin{aligned}
L\left(\mu, \theta, \sigma^{2}\right)= & \sum_{i=1}^{N-1} \ln p\left(r_{i+1} \mid r_{i} ; \mu, \theta, \sigma^{2}\right) \\
= & (N-1) \ln c \\
& +\sum_{i=1}^{N-1}\left\{-u_{i}-v_{i+1}+0.5 q \ln \left(\frac{v_{i+1}}{u_{i}}\right)\right. \\
& \left.\quad+\ln \left[I_{q}\left(2 \sqrt{u_{i} v_{i+1}}\right)\right]\right\} .
\end{aligned}
$$

Finally, we find MLEs of $\mu, \theta$, and $\sigma^{2}$ by maximizing the log-likelihood function (20) over its parameter space:

$$
\left(\widehat{\mu}, \widehat{\theta}, \widehat{\sigma}^{2}\right)=\arg \max _{\left(\mu, \theta, \sigma^{2}\right)} L\left(\mu, \theta, \sigma^{2}\right)
$$

For solving optimization problem (21) we have to rely on numerical solution. The function fminsearch, which is a standard part of MATLAB, makes the job.

3.1.5. MLE for MRJD Model. To implement maximum likelihood estimation, an Euler-Marayuma discretization is performed on (6). In fact, some scholars have considered the problem of estimating the parameters of continuous-time jump processes from discretely sampled data (for reviews and possible solutions, see Cont and Tankov [21] and Weron [22]). Here we follow the approach of Ball and Torous [11] and approximate the model with a mixture of normals. In this setting the intensity $\lambda$ is assumed to be small, so that the arrival rate of two jumps within one period is negligible. Then the Poisson process is well approximated by a simple binary probability $\lambda h$ of a jump and $(1-\lambda) h$ of no jump. Hence, the truncation problem has been solved by adopting a Bernoulli mixture of two normal distributions. It motivates us to approximate the Poisson process in (6) with the Bernoulli process, giving the discretized model:

$$
r_{i}-r_{i-1}=\mu\left(\theta-r_{i-1}\right) h+\varepsilon+Y_{t} J\left(\mu_{J}, \sigma_{J}\right),
$$

where $\varepsilon=\sigma \sqrt{h} \eta, \eta \sim \mathcal{N}(0,1)$, and the parameter $Y_{t}$ is an indicator equal to unity when a jump occurs at a particular time with probability $\lambda$ and zero when there is no jump with probability $1-\lambda$.

Based on (20), we can obtain the density function:

$$
\begin{aligned}
& p\left(r_{i+1} \mid r_{i} ; \mu, \theta, \sigma, \lambda, \mu_{J}, \sigma_{J}\right) \\
& =\lambda \frac{1}{\sqrt{2 \pi\left(\sigma^{2}+\sigma_{J}^{2}\right)}} \exp \left(\frac{-\left(r_{i}-r_{i-1}-\mu\left(\theta-r_{i-1}\right) h-\mu_{J}\right)}{2\left(\sigma^{2}+\sigma_{J}^{2}\right)}\right) \\
& \quad \times(1-\lambda) \frac{1}{\sqrt{2 \pi \sigma^{2}}} \exp \left(\frac{-\left(r_{i}-r_{i-1}-\mu\left(\theta-r_{i-1}\right) h\right)}{2 \sigma^{2}}\right) .
\end{aligned}
$$


Then the MLEs are obtained by maximizing the loglikelihood function with respect to $\mu, \theta, \sigma, \lambda, \mu_{J}$, and $\sigma_{J}$ :

$$
\max _{\mu, \theta, \sigma, \lambda, \mu_{J}, \sigma_{J}} \sum_{i=1}^{N-1} \ln p\left(r_{i+1} \mid r_{i} ; \mu, \theta, \sigma, \lambda, \mu_{J}, \sigma_{J}\right) .
$$

Obviously, the scores are messy nonlinear functions and the maximum likelihood estimates must be computed numerically. Here, we computed the estimates using the optimization toolbox in MATLAB.

3.2. Performance Measures. Now, we have presented five alternative approaches to modeling SHIBOR using stochastic processes. One of the main challenges of the interest rate researches has been comparing different models and selecting the best one. This subsection is designed to answer the important question: What type of process performs best in capturing the behavior of SHIBOR? Actually, there are two classes of model selections: nested in a general model and nonnested models. Log likelihood test can be used to test a model whether it is nested in a more general model. Obviously, the models in previous sections are nonnested in terms of their functional forms. Consequently, here we employ the Vuong test [15] to determine which model is best one.

The classical Vuong test proposed in [15] has been a workhorse for evaluations when the candidate models are nonnested. In fact, the Vuong test compares two models according to the familiar likelihood criterion and leads to probabilistic conclusions about their relative fits. It is well known that the probabilistic feature gives the Vuong test advantage over the various model selection criteria such as the Akaike Information Criterion and the Bayesian Information Criterion which also use the likelihood criterion to compare models. Moreover, Rivers and Vuong [23] extended the approach of Vuong [15] to provide a very general framework for the comparison of some competing dynamic models.

Specifically, the Vuong test considers the average difference in the log likelihoods of two competing statistical models. The null hypothesis of the test is that this average difference is zero. Let $f$ denote model $\mathrm{I}$, which has covariates $\mathbf{X}$ and coefficients $\beta$, and $g$ denotes model II, which has covariates $\mathbf{Z}$ and coefficients $\gamma$. In general, we can write the null hypothesis as

$$
H_{0}: \mathbb{E}_{0}\left[\ln \frac{f(\mathbf{Y} \mid \mathbf{X} ; \beta)}{g(\mathbf{Y} \mid \mathbf{Z} ; \gamma)}\right]=0 .
$$

The null hypothesis simply states that the two models are equally close to the true specification. The expected value in the above hypothesis is unknown. Vuong demonstrates that under fairly general conditions

$$
\frac{1}{N} L R_{N}(\widehat{\beta}, \widehat{\gamma}) \stackrel{\text { a.s. }}{\longrightarrow} \mathbb{E}_{0}\left[\ln \frac{f(\mathbf{Y} \mid \mathbf{X} ; \beta)}{g(\mathbf{Y} \mid \mathbf{Z} ; \gamma)}\right]
$$

where $\operatorname{LR}_{N}(\widehat{\beta}, \widehat{\gamma})$ is the estimated difference in the log likelihoods of the two models.
In fact, when the number of coefficients in two models is different, the Vuong test needs a correction for the degrees of freedom. The adjusted statistic is

$$
\widetilde{L R}_{N}(\widehat{\beta}, \widehat{\gamma})=L R_{N}(\widehat{\beta}, \widehat{\gamma})-K_{N}\left(F_{\theta}, G_{\gamma}\right)
$$

where $K_{N}\left(F_{\theta}, G_{\gamma}\right)$ is the correction factor. Vuong [15] suggests using a correction that corresponds to either Akaike [24] information criteria or Schwarz [25] Bayesian information criteria. Here, we have chosen the latter, making the adjusted statistic,

$$
\widetilde{L R}_{N}(\widehat{\beta}, \widehat{\gamma})=L R_{N}(\widehat{\beta}, \widehat{\gamma})-\left[\left(\frac{p}{2}\right) \ln N-\left(\frac{q}{2}\right) \ln N\right],
$$

where $p$ and $q$ are the numbers of estimated coefficients in models $f$ and $g$, respectively.

Moreover, let us mention that the estimated variance is computed in the usual way (sum of the squares minus square of the sums)

$$
\widehat{\omega}_{N}=\frac{1}{N} \sum_{i=1}^{N}\left[\ln \frac{f(\mathbf{Y} \mid \mathbf{X} ; \widehat{\beta})}{g(\mathbf{Y} \mid \mathbf{Z} ; \widehat{\gamma})}\right]^{2}-\left[\frac{1}{N} \sum_{i=1}^{N} \ln \frac{f(\mathbf{Y} \mid \mathbf{X} ; \widehat{\beta})}{g(\mathbf{Y} \mid \mathbf{Z} ; \widehat{\gamma})}\right]^{2} .
$$

Suitably normalized, the test statistic is normally distributed under the null hypothesis:

$$
\frac{\widetilde{L R}_{N}(\widehat{\beta}, \widehat{\gamma})}{\sqrt{N} \widehat{\omega}_{N}} \stackrel{\mathscr{L}}{\rightarrow} \mathcal{N}(0,1)
$$

where $\stackrel{\mathscr{L}}{\longrightarrow}$ means convergence in distribution and $\mathscr{N}(\cdot, \cdot)$ denotes the normal distribution.

The Vuong test can be described in simple terms. If the null hypothesis is true, the average value of the log-likelihood ratio should be zero. If $f$ is better than $g$, the average value of the log-likelihood ratio should be significantly greater than zero. If the reverse is true, the average value of the loglikelihood ratio should be significantly less than zero. In other words, the Vuong test statistic is simply the average loglikelihood ratio suitably normalized. In general, this model selection can be used to compare different models estimated using the MLE method.

\section{Empirical Results}

In this section, for an illustration of the method derived in previous sections, we apply these approaches to the real data. We model the term structure of SHIBOR in China and propose estimation of the implied parameters using the approaches described above. Moreover, we will infer the best model among the proposed models based on the test of Vuong [15].

4.1. Data Description. In January 2007, China established SHIBOR System with the aim of building a benchmark yield curve. The SHIBOR is the average interest rate at which term deposits are offered between prime banks in the Shanghai 


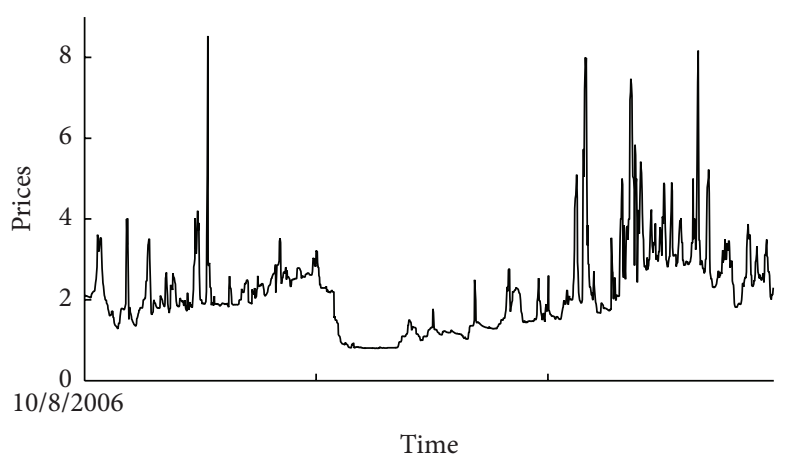

(a) Daily closing prices

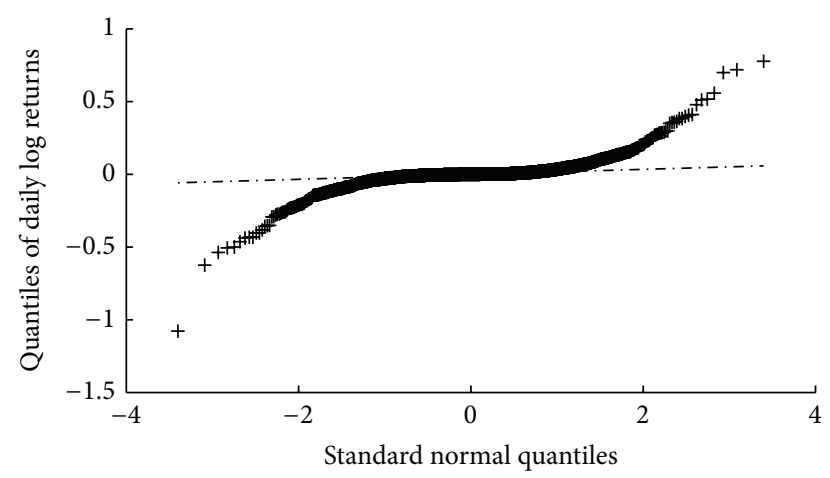

(c) Q-Q plot

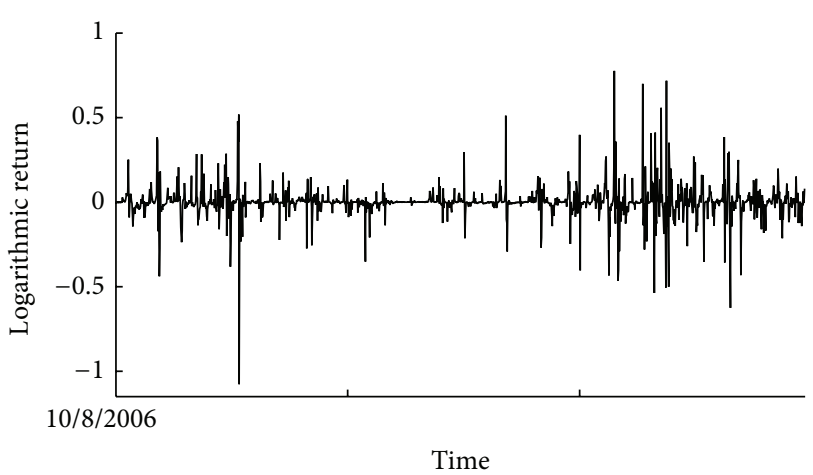

(b) Daily log returns

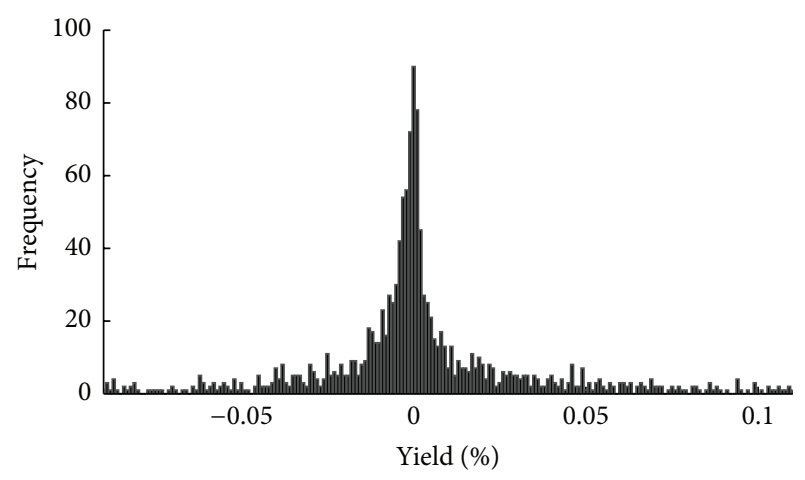

(d) Probability density of the return

FIGURE 1: Some statistical figures of daily returns for the O/N SHIBOR rate from October 8th 2006 to September 10 th 2012.

wholesale money market or interbank market. In fact, the SHIBOR rate is not determined in a funding market but is set in a similar way to LIBOR, with the rate calculated as an arithmetic average of Renminbi offered rates by participating banks (currently 16) and is a fixing at 11:30 a.m. on each business day. The SHIBOR is the average interest rate at which term deposits are offered between prime banks in the Shanghai wholesale money market or interbank market. In fact, three key short-term interbank interest rates, namely, China Interbank Offered Rate, SHIBOR, and the repo rate, have followed each other very closely over the past few years, with volatility having increased substantially since late 2005. Given the greater liquidity in the repo market (with the turnover in the repo market far exceeding that in the uncollateralized China Interbank Offered Rate market) and the tight relationship between the SHIBOR and the China Interbank Offered Rate market rates with the repo, it seems little additional information is added by the SHIBOR rates for maturities and period when trading is active in either the interbank repo or loan market. The SHIBOR system does, however, provide a benchmark interest rate quote when interbank trading is limited.

It is well known that SHIBOR is calculated, announced, and named on the technological platform of the National Interbank Funding Center in Shanghai. It is a simple, noguarantee, wholesale interest rate calculated by arithmetically averaging all the interbank Renminbi lending rates offered by the price quotation group of banks with a high credit rating. Currently, the SHIBOR consists of eight maturities: overnight, 1 week, 2 weeks, 1 months, 3 months, 6 months, 9 months, and 1 year. The price quotation group of SHIBOR consists of 16 commercial banks. These quoting banks are primary dealers of open market operation or market makers in the FX market, with sound information disclosure and active Renminbi transactions in China's money market. SHIBOR Working Group of PBC decides and adjusts the panel banks, supervises and administrates the SHIBOR operation, and regulates the behavior of the quoting banks and the specified publisher in accordance with the Implementation Rules of SHIBOR.

Actually, the data utilized in our empirical study are extracted from the web of http://www.shibor.org/shibor/ web/DataService.jsp. Our study focuses on the time series of $\mathrm{O} / \mathrm{N}, 1$-week, 2-week, and 1-month SHIBOR in China spanning from October 8th 2006 to October 10th 2012, forming 1485 observations of 4 time series. Let $r_{(\cdot)}$ be the daily observations. Then the index prices are observed at time interval of $h=1 / 250$ (e.g., data collected once a day) and the returns are calculated using the logarithmic differenced data.

Let us now turn to the analysis of real financial data. As explained above, four indices are transformed into the log-return format. Basic descriptive plots for SHIBOR are presented in the following figures. Figure 1 provides some empirical data about the $\mathrm{O} / \mathrm{N}$ of SHIBOR in China: Figure 1(a) shows the daily closing values of the $\mathrm{O} / \mathrm{N}$ of SHIBOR in the sample period. There appears no long-run average level 


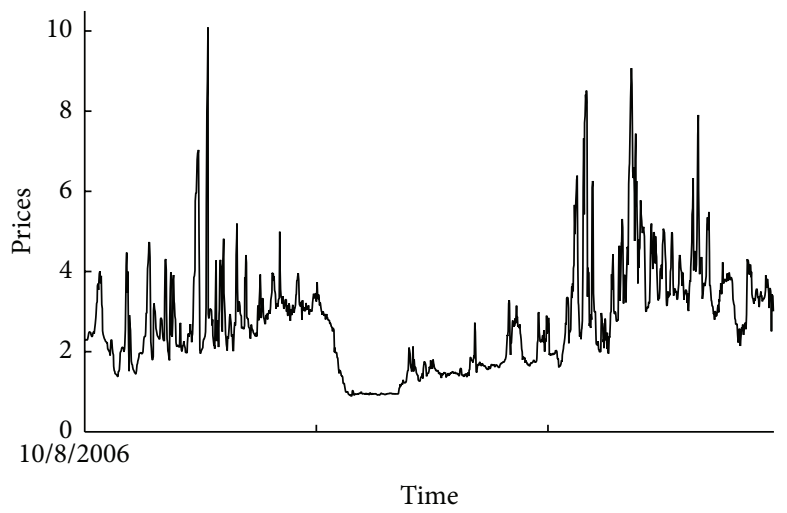

(a) Daily closing prices

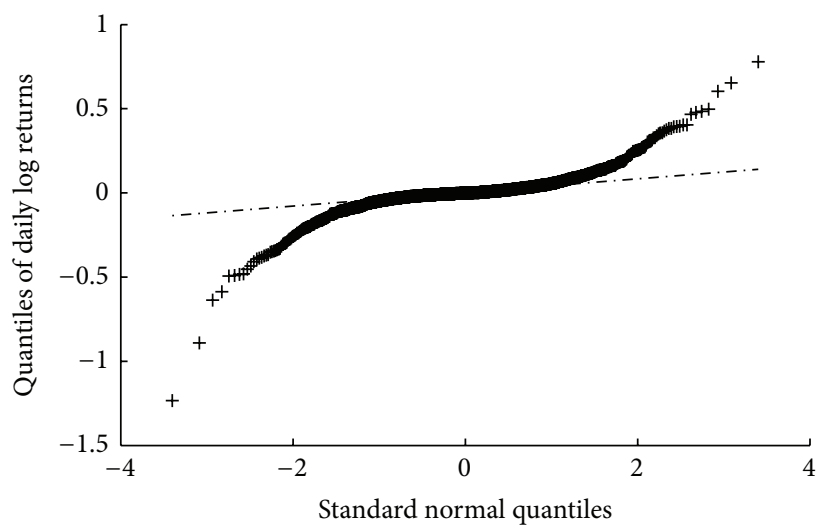

(c) Q-Q plot

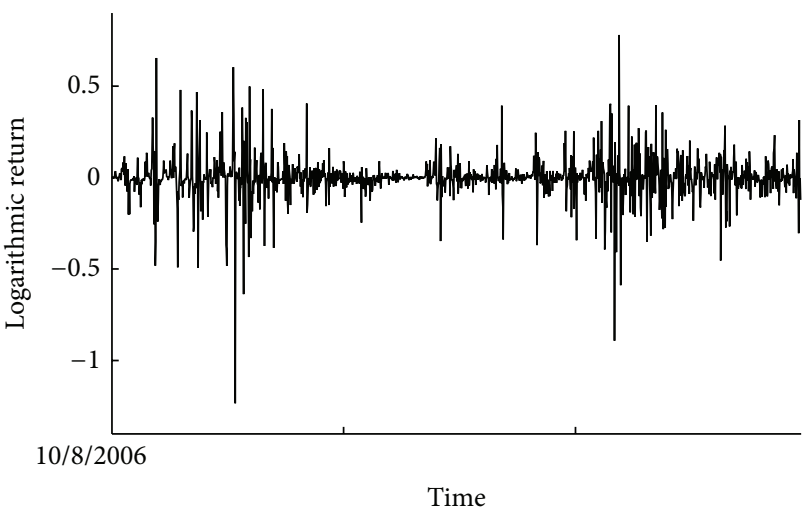

(b) Daily log returns

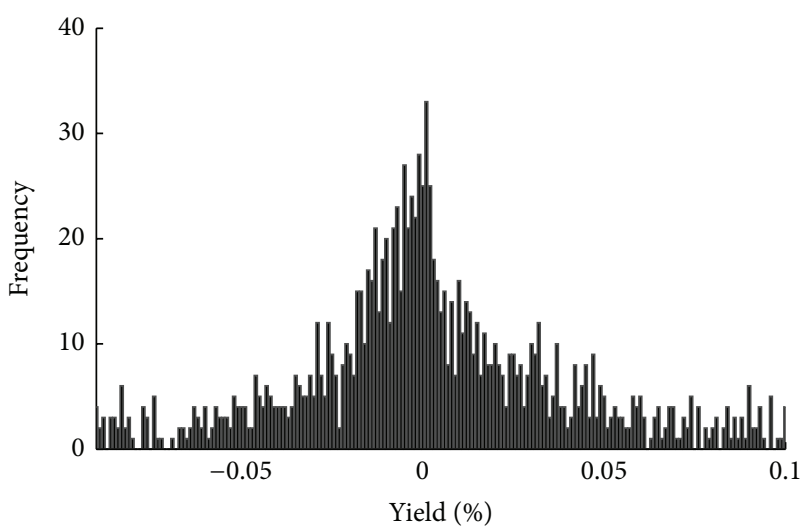

(d) Probability density of the return

FIGURE 2: Some statistical figures of daily returns for the 1-week SHIBOR rate from October 8th 2006 to September 10 th 2012.

about which the series evolves. Figure 1(b) illustrates the continuously compounded returns (the log returns) associated with the price series in Figure 1(a). It illustrates the clustering effect of compounded returns. In other words, large changes tend to be followed by large changes - of either sign - and small changes tend to be followed by small changes. Figure $1(\mathrm{c})$ presents the quantile-quantile (hereafter $\mathrm{Q}-\mathrm{Q}$ ) figure during the sample period. In fact, the Q-Q plot is an illustration of how strong the deviations from normality are on the tails of the distribution. Finally, Figure 1(d) depicts the empirical probability density function of the $\mathrm{O} / \mathrm{N}$ of SHIBOR. As implied by the positive excess kurtosis, the empirical density has a higher central peak and "fat tails From the evidence of a highly peaked and fat-tailed distribution, we can conclude that small and large movements in the empirical samples occur more likely compared to normally distributed log returns. Similarly, some statistical figures of 1-week, 2-week, and 1-month SHIBOR are shown in Figures 2-4, respectively.

To give a brief insight into the properties of the selected data, Table 1 tabulated the basic descriptive statistics of $\mathrm{O} / \mathrm{N}$, 1-week, 2-week, and 1-month SHIBOR in the sample period. Names are given in the first column. The second, third, fourth, and fifth columns contain the basic descriptive statistics for these indices. Moreover, both skewness and kurtosis are also presented in this table. The largest historical return over the full period is realized by 1 month, followed by 2 weeks. The lowest average over the full period is found for $\mathrm{O} / \mathrm{N}$. The most volatile one is 1 week. Now, let us look at the skewness. The skewness of a symmetric distribution, such as the normal distribution, is zero. However, none of the series seems to be symmetric. All the series have negative skewness, which implies that the distributions have a long left tail. The next statistic that is important for our analysis is the kurtosis. In our case, both series have a kurtosis that exceeds a value of three, which is the kurtosis of the normal distribution. This means that the distributions are peaked (leptokurtic) relative to the normal distribution. Because both descriptive statistics (skewness and kurtosis) indicate deviations from normal values, we can expect that the observed distributions are not normally distributed. The results are also confirmed by the Q-Q plots (see Figures 1(c), 2(c), 3(c), and 4(c)). As we can see from Figures 1(c), 2(c), 3(c), and 4(c), the plots indicate an S-shaped curve, which is a typical sign of a nonnormal distribution in a financial time series.

The basic stylized facts are as follows: near nonstationary behavior (slow mean reversion) can be observed; large changes and small changes are clustered together; the volatility increases with the level (level effect); obviously larger skewness and great kurtosis indicate high peaks and 


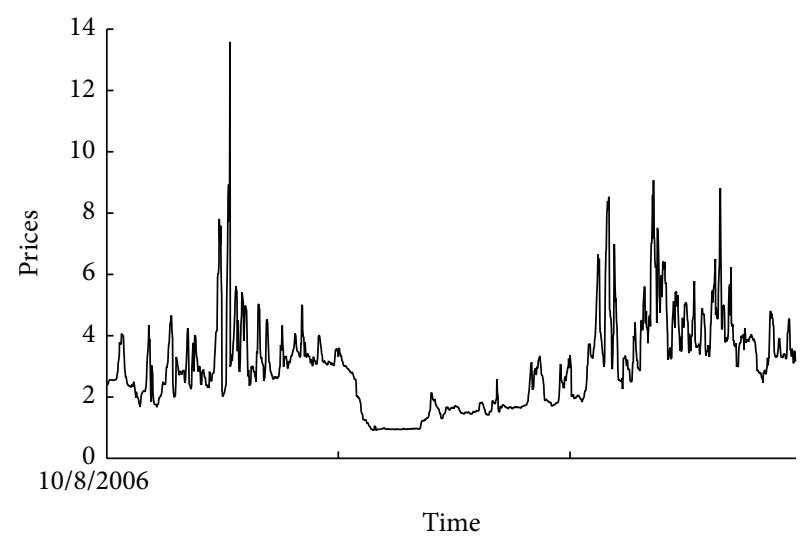

(a) Daily closing prices

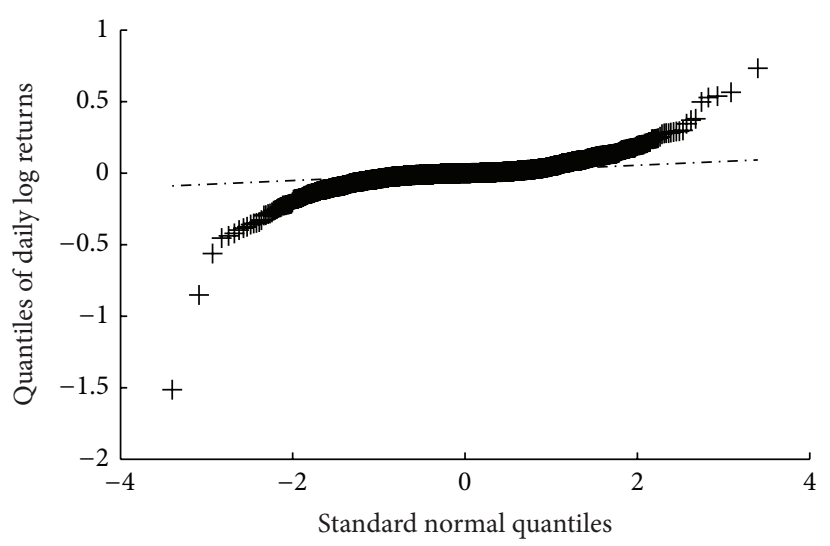

(c) Q-Q plot

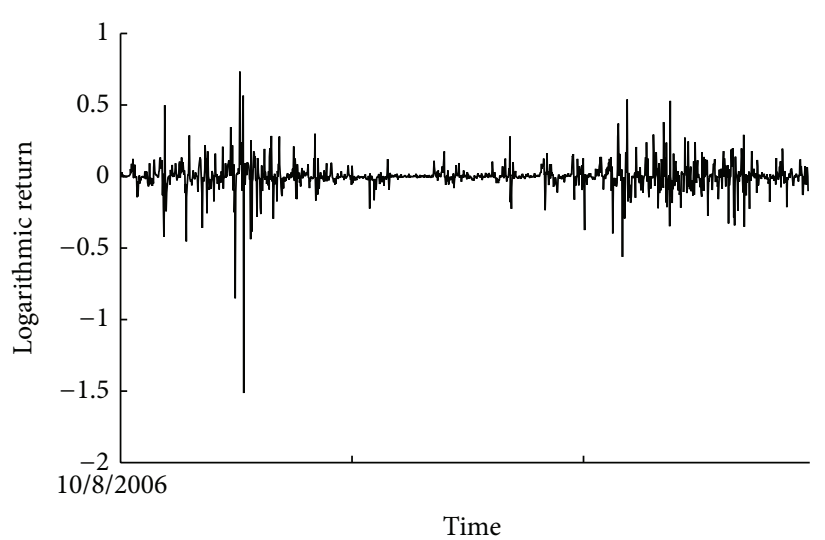

(b) Daily $\log$ returns

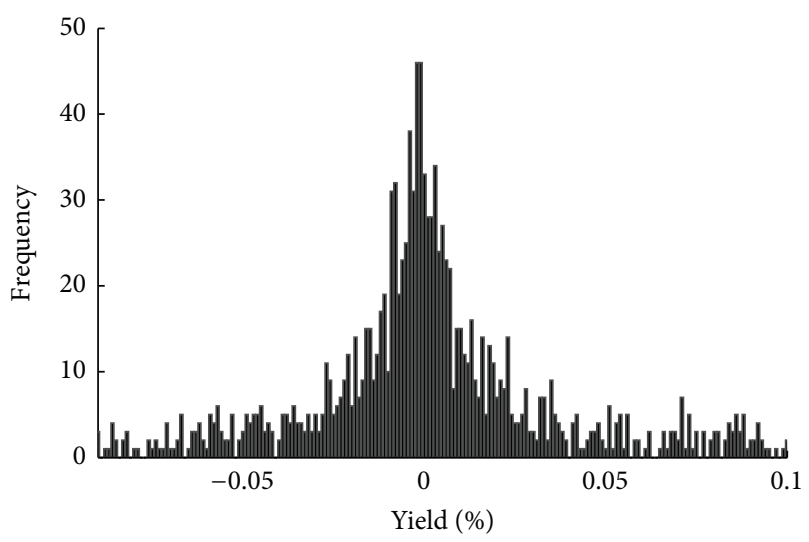

(d) Probability density of the return

FIGURE 3: Some statistical figures of daily returns for the 2-week SHIBOR rate from October 8th 2006 to September 10 th 2012.

TABLE 1: Summary statistics of daily returns for time series of the return of SHIBOR.

\begin{tabular}{|c|c|c|c|c|c|c|}
\hline Index & Minimum & Maximum & Mean & Std. Dev. & Skewness & Kurtosis \\
\hline $\mathrm{O} / \mathrm{N}$ & -1.0782 & 0.7759 & $5.5152 e-005$ & 0.0972 & -0.2589 & 26.9025 \\
\hline 1 week & -1.2333 & 0.7795 & $1.8154 e-004$ & 0.1127 & -1.0119 & 22.0629 \\
\hline 2 weeks & -1.5153 & 0.7323 & $1.8669 e-004$ & 0.0969 & -2.7083 & 53.9854 \\
\hline 1 month & -0.7899 & 0.2847 & $2.5266 e-004$ & 0.0478 & -4.5803 & 82.1696 \\
\hline
\end{tabular}

fat tails (leptokurtic). Hence, a pronounced feature of these data is that the SHIBOR is more volatile.

4.2. Estimation Results and Model Comparisons. Based on the situation of discrete observations mentioned in the previous sections, we now proceed to estimate all the unknown parameters of stochastic models mentioned above based on the combination of the maximum likelihood approach and the selected data. Table 2 reports the parameter estimates for discretized single-factor diffusion models and log-likelihood value.

As shown, the models vary in their explanatory power for interest rate changes. These estimations reveal some important stylized facts of SHIBOR. There exists significant mean reversion in SHIBOR. For example, the estimates of the drift parameters in Vasicek, CIR, and MRJD models in
Table 2 all show significant mean reversion. Moreover, the log-likelihood value increases from -186.20861 to -13.5398 by introducing jump diffusion model. In fact, the contribution of the introduction of jump is significant. In Table 2, the loglikelihood value of MRJD increases to -13.5398 when we use a MRJD.

Comparing the log likelihoods from Table 2, we see that MRJD appears to fit better, although we do not know whether this difference is meaningful or not. To answer this question, the Vuong test has been used for comparison of models of Merton, GBM, Vasicek, CIR, and MRJD. These results are presented in Table 3. Here, the desired level of significance is 1.959964. The results of the tests leave no room for ambiguity: the Vuong test is in favor of the MRJD model. Thus, we can be confident that jumps are an essential component of interest rate models. 


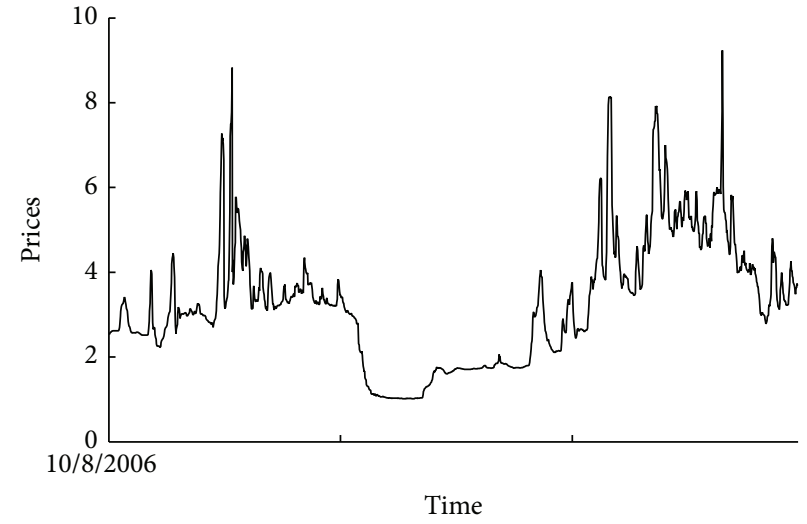

(a) Daily closing prices

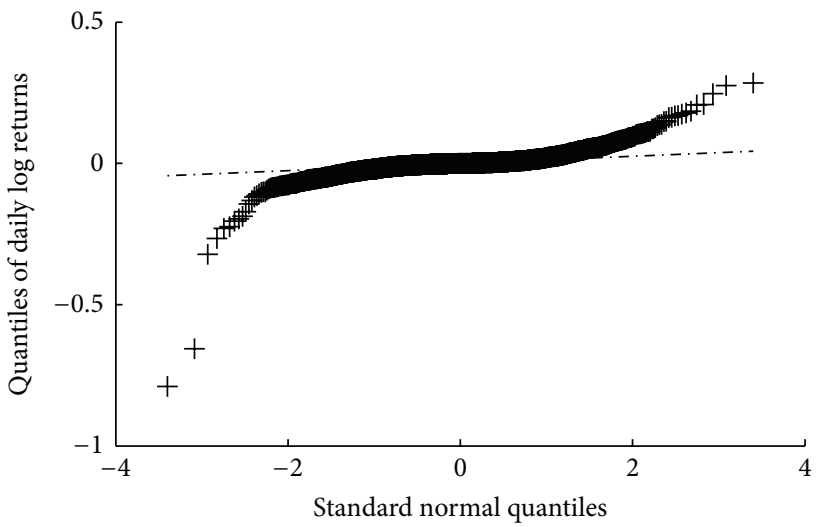

(c) Q-Q plot

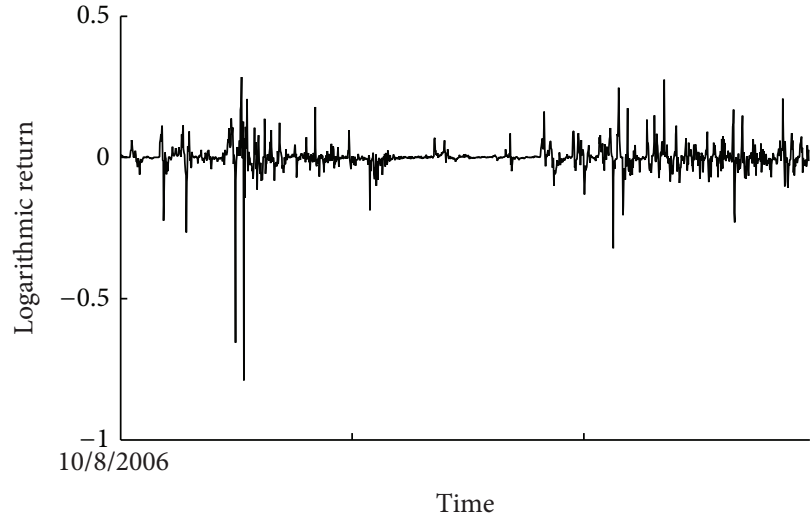

(b) Daily log returns

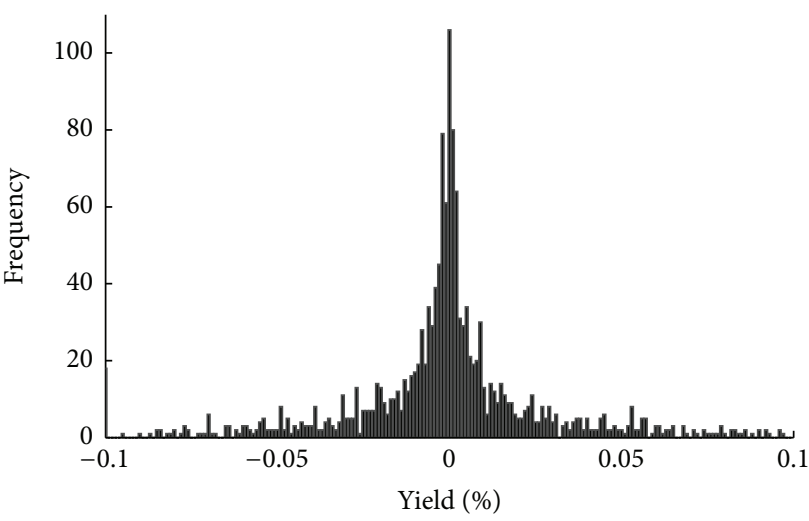

(d) Probability density of the return

FIGURE 4: Some statistical figures of daily returns for the 1-month SHIBOR rate from October 8th 2006 to September 10 th 2012.

TABLE 2: Parameter estimates for the single-factor diffusion models.

\begin{tabular}{|c|c|c|c|c|c|c|c|c|}
\hline \multirow{2}{*}{ Series } & \multirow{2}{*}{ Model name } & \multicolumn{6}{|c|}{ Parameters } & \multirow{2}{*}{ Log likelihood } \\
\hline & & $\mu$ & $\sigma$ & $\theta$ & $\lambda$ & $\mu_{J}$ & $\sigma_{J}$ & \\
\hline \multirow{5}{*}{$\mathrm{O} / \mathrm{N}$} & Merton & 0.386810 & 0.366409 & - & - & - & - & -116.5897 \\
\hline & GBM & 0.004838 & 0.102661 & - & - & - & - & -79.4846 \\
\hline & Vasicek & 0.059131 & 0.357140 & 2.182412 & - & - & - & -67.1018 \\
\hline & CIR & 0.185532 & 0.248036 & 5.304625 & - & - & - & -38.2561 \\
\hline & MRJD & 0.002212 & 0.045752 & 2.001096 & 0.054475 & -0.047486 & 0.745071 & -16.3986 \\
\hline \multirow{5}{*}{ 1-Week } & Merton & 0.505131 & 0.452737 & - & - & - & - & -108.7844 \\
\hline & GBM & 0.006433 & 0.113952 & - & - & - & - & -93.3939 \\
\hline & Vasicek & 0.058609 & 0.442345 & 2.732211 & - & - & - & -86.9711 \\
\hline & CIR & 0.043466 & 0.219753 & 2.735201 & - & - & - & -58.2525 \\
\hline & MRJD & 0.003102 & 0.147420 & 2.408793 & 0.035441 & -0.147026 & 1.165968 & -13.5398 \\
\hline \multirow{5}{*}{ 2-Week } & Merton & 0.529391 & 0.491417 & - & - & - & - & -186.2086 \\
\hline & GBM & 0.046521 & 0.093887 & - & - & - & - & -106.2416 \\
\hline & Vasicek & 0.058597 & 0.451624 & 2.731857 & - & - & - & -105.7956 \\
\hline & CIR & 0.027683 & 0.211341 & 2.803126 & - & - & - & -68.6953 \\
\hline & MRJD & 0.005202 & 0.210520 & 2.112525 & 0.042165 & -0.22365 & 1.236651 & -13.8344 \\
\hline \multirow{5}{*}{ 1-Month } & Merton & 0.619768 & 0.252719 & - & - & - & - & -164.5811 \\
\hline & GBM & 0.013203 & 0.044481 & - & - & - & - & -88.6862 \\
\hline & Vasicek & 0.013835 & 0.243282 & 3.313312 & - & - & - & -56.6224 \\
\hline & CIR & 0.080097 & 0.105921 & 3.354525 & - & - & - & -37.7186 \\
\hline & MRJD & 0.001618 & 0.078245 & 2.000189 & 0.038285 & 0.023883 & 0.714316 & -27.5073 \\
\hline
\end{tabular}


TABLE 3: Results of the Vuong test.

\begin{tabular}{lccccc}
\hline & Merton & GBM & Vasicek & CIR & MRJD \\
\hline Merton & - & 4.180489 & 2.241832 & 4.799925 & 4.317839 \\
GBM & - & - & 3.370632 & 3.110825 & 3.799735 \\
Vasicek & - & - & - & 2.012456 & 2.482186 \\
CIR & - & - & - & - & 3.302152 \\
MRJD & - & - & - & - & - \\
\hline
\end{tabular}

\section{Conclusions}

Continuous-time models of the term structure of interest rates developed so far use a stochastic process in order to model the dynamics of the short-term interest rate. While there is a great deal of interest in using stochastic differential equations to model financial time series data, it has been difficult to find effective ways to estimate these models. An extensive collection of continuous-time models of the shortterm interest rate is evaluated over data sets of mature markets that have appeared previously in the literature. However, due to the relatively short history of the Chinese bond markets and the strict regulation of Chinese interest rates, there has been little study of spot interest rates in China.

Despite the numerous empirical studies of spot interest rate models, litter effort has been denoted to examining the behavior of SHIBOR. In this paper, we have contributed to literature by providing the comprehensive empirical analysis of SHIBOR. We propose the maximum likelihood methodology to estimate the parameters of five single-factor interest rate models and compare these five different models of shortterm interest rate dynamics in order to determine which model best fits the SHIBOR data. In addition to the wellknown optimality properties of MLE, the availability of the likelihood provides a convenient tool for specification analysis. The results of the Vuong test show that the popular models, including Merton, GBM, Vasicek, CIR, and MRJD, perform poorly relative to continuous models. The MRJD should be preferred other five models.

There are a couple of issues that hold potential for future research in this area. First, we must acknowledge that the single factor model used here, though empirically tractable, is also subject to some criticism. The dependence on a single factor greatly limits the possible shapes of the yield curve and often leads into situations, where the theoretical yield curve does not correspond to the market yield curve. Consequently, the first suggestion is to introduce the twofactor model or the three-factor model. Although regime switching and jumps are important for modeling Chinese spot rate dynamics, they are still grossly misspecified. There is a long way to go before we reach a correct specification for Chinese spot rate dynamics. Hence, the second suggestion is to explore possible sources of model misidentification by examining the marginal distribution and model dynamics separately. Finally, one of the main assumptions of Vuong's model selection criterion is that the data are independent and identically distributed, which normally does not hold for time series. Thus the third direction is to present some modified tests of the classical Vuong test.

\section{Conflict of Interests}

The author declares that he has no financial and personal relationships with other people or organizations that can inappropriately influence his work, there is no professional or other personal interest of any nature or kind in any product, service, and/or company that could be construed as influencing the position presented in, or the review of, the paper.

\section{Acknowledgments}

This research was supported by the National Natural Science Foundation of China (nos. 71101056 and 71301144), the Major Program of National Social Science Foundation of China (11\&ZD156), the Humanity and Social Science Youth foundation of Ministry of Education of China (no. 13YJC630227), the Zhejiang Provincial Natural Science Foundation of China (no. LQ13G010001), and the Educational Commission of Zhejiang Province of China (no. Y201329832).

\section{References}

[1] F. Black and M. Scholes, "The pricing of options and corporate liabilities," Journal of Political Economy, vol. 81, no. 3, pp. 637654, 1973.

[2] R. Merton, “Theory of rational option pricing," Bell Journal of Economics, vol. 4, no. 1, pp. 141-183, 1973.

[3] O. Vasicek, "An equilibrium characterization of the term structure," Journal of Financial Economics, vol. 5, no. 2, pp. 177-188, 1977.

[4] J. C. Cox, J. E. Ingersoll, and S. A. Ross, "A theory of the term structure of interest rates," Econometrica, vol. 53, no. 2, pp. 385407, 1985.

[5] K. C. Chan, G. A. Karolyi, F. A. Longstaff, and A. B. Sanders, "An empirical comparison of alternative models of the short-term interest rate," Journal of Finance, vol. 47, no. 3, pp. 1209-1227, 1992.

[6] Y. Aït-Sahalia, "Testing continuous-time models of the spot interest rate," Review of Financial Studies, vol. 9, no. 2, pp. 385426, 1996.

[7] S. R. Das, “The surprise element: jumps in interest rates," Journal of Econometrics, vol. 106, no. 1, pp. 27-65, 2002.

[8] B. Eraker, M. Johannes, and N. Polson, "The impact of jumps in volatility and returns," Journal of Finance, vol. 58, no. 3, pp. 1269-1300, 2003.

[9] M. Johannes, "The statistical and economic role of jumps in continuous-time interest rate models," Journal of Finance, vol. 59, no. 1, pp. 227-260, 2004.

[10] R. C. Merton, "Option pricing when underlying stock returns are discontinuous," Journal of Financial Economics, vol. 3, no. 1-2, pp. 125-144, 1976.

[11] C. Ball and W. Torous, "A simplified jump process for common stock returns," Journal of Financial and Quantitative Analysis, vol. 18, no. 1, pp. 53-65, 1983.

[12] S. G. Kou, "A jump-diffusion model for option pricing," Management Science, vol. 48, no. 8, pp. 1086-1101, 2002.

[13] Y. Hong, H. Lin, and S. Wang, "Modeling the dynamics of Chinese spot interest rates," Journal of Banking and Finance, vol. 34, no. 5, pp. 1047-1061, 2010. 
[14] W. Xiao, W. Zhang, and W. Xu, "Parameter estimation for fractional Ornstein-Uhlenbeck processes at discrete observation," Applied Mathematical Modelling, vol. 35, no. 9, pp. 4196-4207, 2011.

[15] Q. H. Vuong, "Likelihood ratio tests for model selection and non-nested hypotheses," Econometrica, vol. 57, no. 2, pp. 307333, 1989.

[16] T. Marsh and E. Rosenfeld, "Stochastic processes for interest rates and equilibrium bond prices," Journal of Finance, vol. 38, no. 2, pp. 635-646, 1983.

[17] K. Lim, H. Ting, M. Warachka et al., "The implied jump risk of libor rates," Journal of Banking and Finance, vol. 29, no. 10, pp. 2503-2522, 2010.

[18] W. Wang, F. Ding, and J. Dai, "Maximum likelihood least squares identification for systems with autoregressive moving average noise," Applied Mathematical Modelling, vol. 36, no. 5, pp. 1842-1853, 2012.

[19] Y. Hu, D. Nualart, W. Xiao, and W. Zhang, "Exact maximum likelihood estimator for drift fractional Brownian motion at discrete observation," Acta Mathematica Scientia, vol. 31, no. 5, pp. 1851-1859, 2011.

[20] W. Feller, "Two singular diffusion problems," Annals of Mathematics, vol. 54, no. 1, pp. 173-182, 1951.

[21] R. Cont and P. Tankov, Financial Modelling with Jump Processes, Chapman \& Hall, 2004.

[22] R. Weron, Modeling and Forecasting Electricity Loads and Prices: A Statistical Approach, Wiley, Chichester, UK, 2006.

[23] D. Rivers and Q. Vuong, "Model selection tests for nonlinear dynamic models," Econometrics Journal, vol. 5, no. 1, pp. 1-39, 2002.

[24] H. Akaike, "Information theory and an extension of the maximum likelihood principle," in 2nd International Symposium on Information Theory, vol. 1, pp. 267-281, Akademiai Kiado, 1973.

[25] G. Schwarz, "Estimating the dimension of a model," Annals of Statistics, vol. 6, no. 2, pp. 461-464, 1978. 


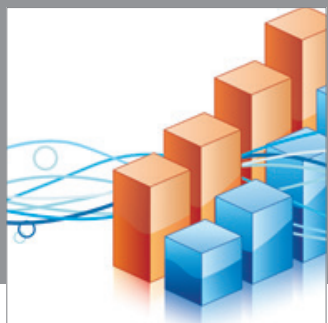

Advances in

Operations Research

mansans

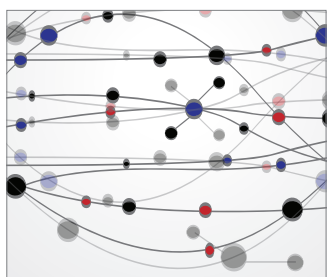

The Scientific World Journal
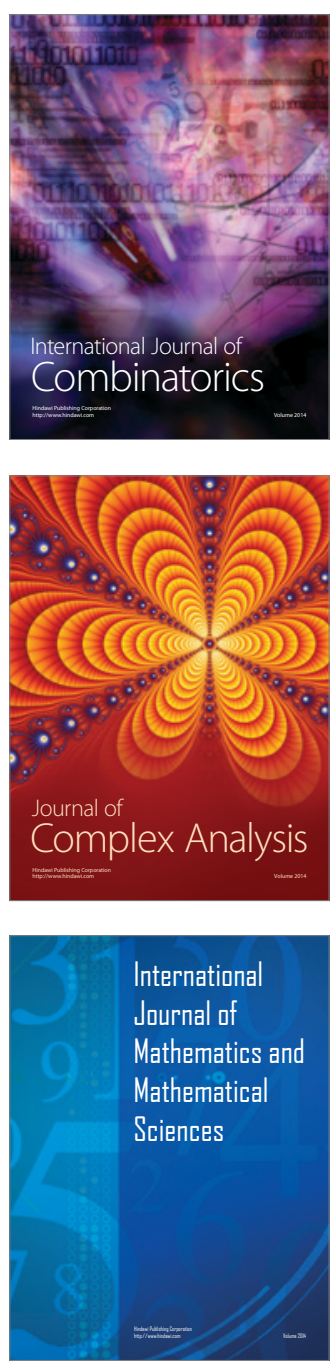
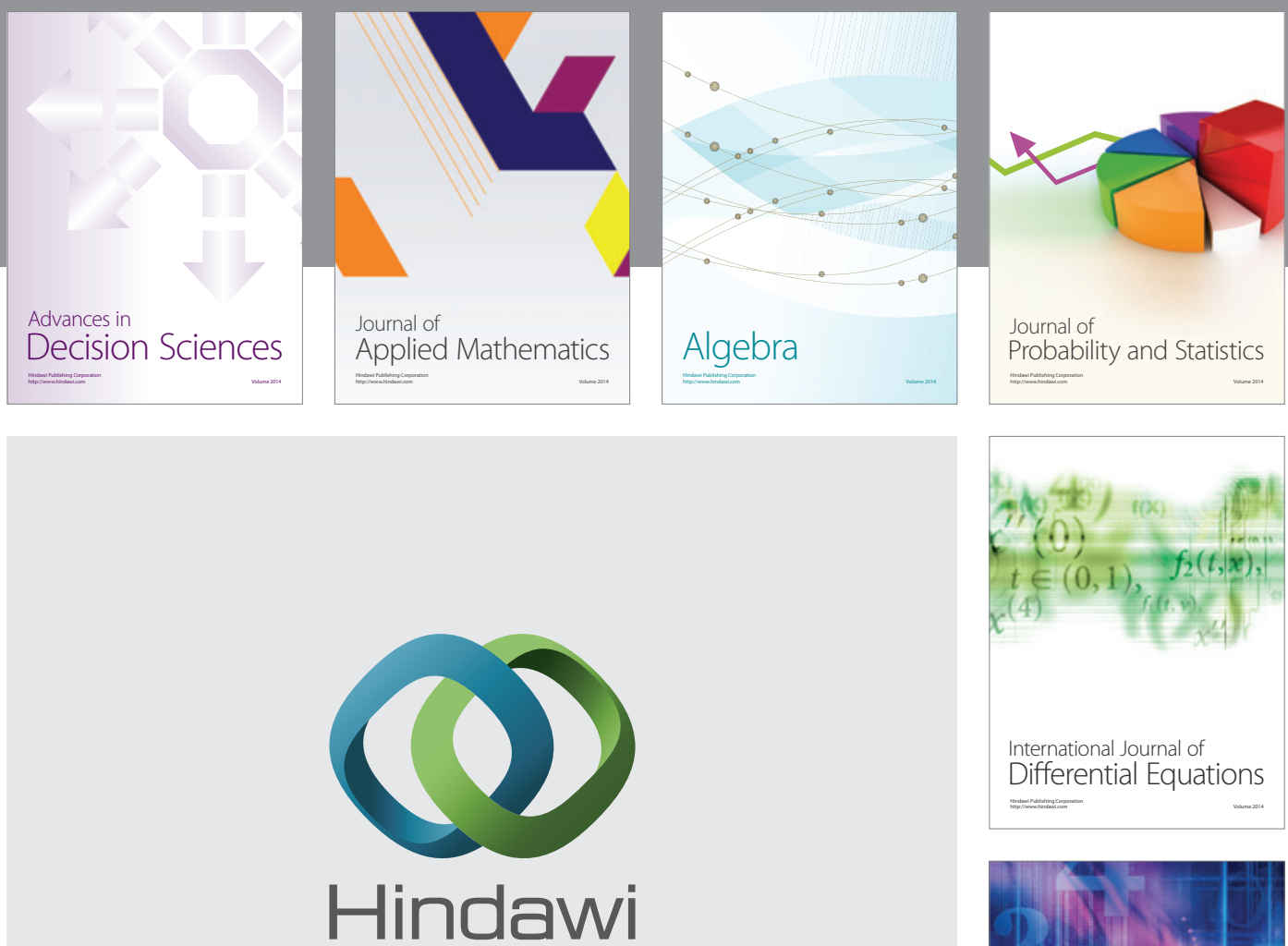

Submit your manuscripts at http://www.hindawi.com
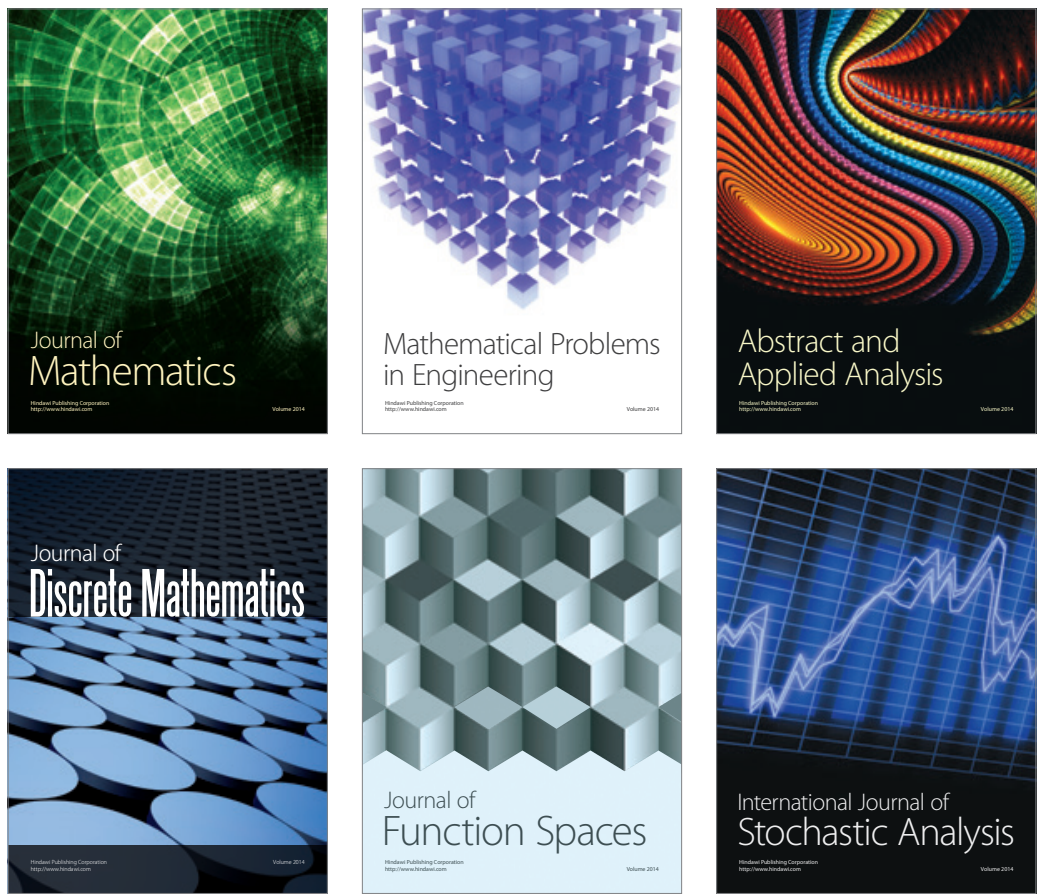

Journal of

Function Spaces

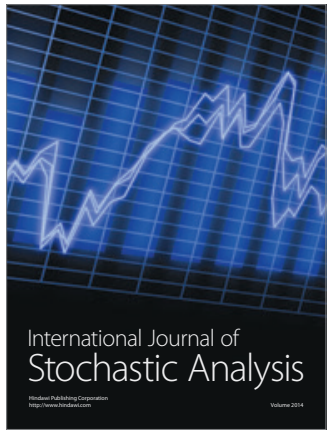

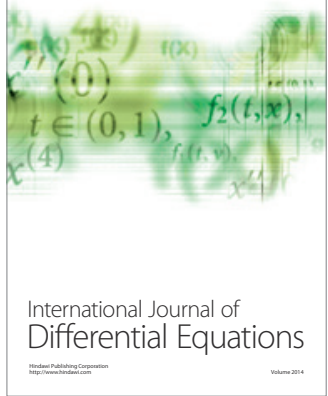
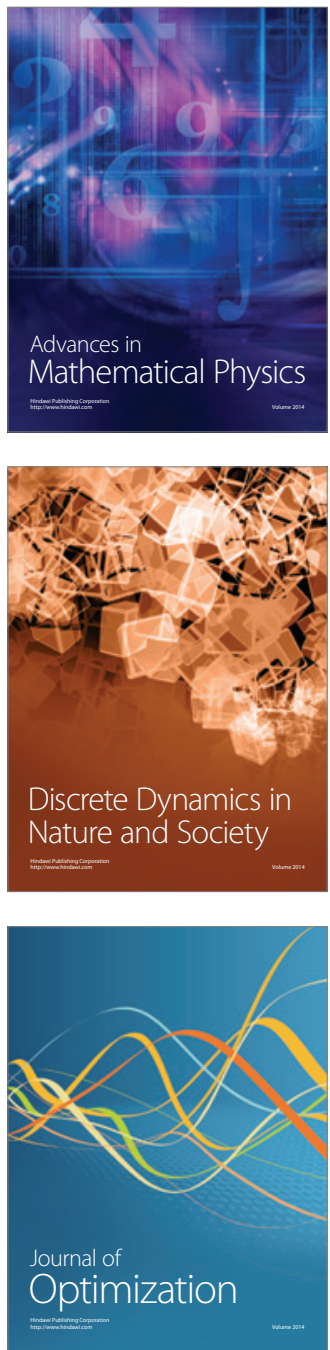